International Mathematical Forum, 1, 2006, no. 12, 577 - 591

\title{
Modelling Conceptual Change in Physics using Probabilistic, Fuzzy and Symbolic Approaches
}

\author{
M. A. Symeonaki \\ Department of Social Politics, Panteion University, \\ 136 Syggrou Av, 176 71, Athens, Greece \\ msymeon@unipi.gr,msimeon@panteion.gr
}

\begin{abstract}
The present paper reports on simulation of conceptual change that concerns the development of the concept of force. Forty nine elementary school students were given three drawings depicting objects of various sizes in different kinetic states or being pushed by a human agent. They were asked whether a force was being exerted on the objects and to explain why. The same questions were posed to the same students after eight weeks. During this period of eight weeks the students participated in a special instruction in mechanics, which emphasised on the concept of force. The dynamic evolution of the system is studied with the aid of Markov systems. A fuzzy expert system is given in order to deal with the uncertainty introduced in the estimation of the transition probabilities due to lack of data and measurement errors. The proposed hybrid (probabilistic - fuzzy) system provides useful information for studying conceptual change and students' understanding.
\end{abstract}

Keywords: mental models, conceptual change, population dynamics, Markov systems, fuzzy logic, PROLOG

\section{Introduction}

This paper is concerned with modelling conceptual change in Physics using fuzzy Markov systems and symbolic approaches. The purpose and contribution of the present paper is to study the dynamic evolution of the population system consisting of 49 
elementary school students, in concern with the mental models the pupils use and provide tools for the simulation of conceptual change concerning the concept of force. The study of conceptual change is one of the most engaging issues in Cognitive Science. A number of researchers (Vosniadou and Brewer [1992, 1994], Muehlenbrock [1994a, 1994b], Morik [1996], Vosniadou, Champesme, Kasyer et al. [2000], Ioannides and Vosniadou [1993], Vosniadou [1994], Clement [1983], McCloskey [1983], Carey [1991]) have proposed theories that analyze the development of knowledge acquisition in different domains.

The present work differs both in the type of results we obtain and in the techniques we use. First, rather that symbolic programming we use Fuzzy Markov systems developed by Symeonaki et al [2000, 2002] and used (see, e.g., Symeonaki and Stamou [2002a, 2002b] in the analysis of Markov population systems where the estimation of the transition probabilities is complex due to lack of data.

We are motivated largely by problems associated with the dynamic evolution and the asymptotic behaviour of population systems consisting of students in concern with conceptual change. Analysis and design of complex systems often involve two kinds of uncertainty: randomness and fuzziness. The randomness models stochastic variability and fuzziness models measurement imprecision due to linguistic structure or incomplete information ( $\mathrm{Hu}$ et al [2000]). The application of fuzzy reasoning in the population system provides us with an effective tool in the effort to model conceptual change, since it gives the power of using symbolic heuristic knowledge that the experts possess. Therefore, one is able to study and find in a closed analytical form the asymptotic behaviour of the system and confirm that there is apparently a high future concentration to the most scientific models.

The paper is organised as follows. In Section 2 we shortly describe the results of previous work on the study of conceptual change concerning the concept of force and analyze the conceptual framework we use. In Section 3 a full description of the population system used is provided. The theory of fuzzy logic and fuzzy reasoning is combined with the theory of Markov systems and the concept of a fuzzy Markov system is used. 


\section{Problem Statement}

Most scientists studying conceptual change now agree that by the time most students start school, they have already constructed considerable knowledge of the physical world that surrounds them, on the basis of their everyday experiences. This knowledge acquisition process takes place before they come in contact with systematic teaching at school. Vosniadou and Brewer [1994] have developed a theoretical background of the way that this knowledge develops in the scientific field of Astronomy. Moreover, Vosniadou [1994] has proposed that the generation of a conceptual system for the interpretation of observations of the physical world starts early in infancy and concludes into a relatively established frame theory for the physical world when children start going to school. Ioannides and Vosniadou [1993] used the theoretical model and methodology described in Vosniadou and Brewer [1992, 1995] and Vosniadou [1994] in order to investigate the development of the concept of force. The results showed that it is possible to classify approximately $80 \%$ of the students in the sample as using one of the following mental models:

- Force in inanimate objects - Force is an inherent (related to physical characteristics) or an acquired (transferred to the inanimate object through direct contact with another object) property of objects:

1. M1: force is an internal property of objects and it is related to their size or weight.

2. M4: there is an internal force in objects, which is affected by the size/weight and by the position-movement (force is being exerted to the object, only if the object moves).

- Force in animate objects - Force is a property of animate objects related to physical characteristics, such as size, weight, etc:

1. A1: force is exerted if a person pushes or tries to push (the outcome of the push is irrelevant).

2. A2: force is exerted if a person pushes very hard (the outcome of the push is relevant, since if the object does not move it means that the person has not tried enough).

3. A3: force is exerted if a person pushes very hard and consumes energy (the outcome of the push is irrelevant). 
4. A4: force is exerted if a person tries and the object moves (the outcome of the push is relevant).

- Moreover, the following models have appeared:

1. $\mathbf{P}:$ The force of gravity is exerted.

2. $\mathbf{A}+\mathbf{P}:$ The force of gravity and the force of man are exerted.

3. P+AP: The force of gravity and the atmospheric pressure are exerted.

4. F: The force of friction is exerted.

In later studies (Vosniadou et al [2000]) the conceptual framework introduced in Vosniadou and Brewer [1992, 1994] was used in order to provide a psychological process model of the solution of mechanics problems by elementary school students. The subjects of the study were forty nine $5^{\text {th }}$ grade students of elementary school. The questions used were three, they concerned the concept of force and they were posed to the pupils twice. Between these two times (which covered a period of 8 weeks) the students participated in a special instruction course in mechanics. More specifically, the questions used are stratified into the following categories:

1. Questions that concern stationary objects.

2. Questions that concern stationary objects being pushed by a human agent.

3. Questions that concern objects that move, when pushed by a human agent.

More precisely, the questions used were the following:

\section{Question 1:}

"Two stones, one big and one small are stationary on the ground. Is there a force exerted on the stones?"

\section{Question 2:}

"A person is pushing two stones, one big and one small. He/she does not succeed in moving the first one, whereas the second one moves. Is there a force exerted on the stones?”

\section{Question 3:}

"A person pushes the box, but does not succeed in moving it. Is there a force exerted on the box?"

The present paper brings together the know-how from the fields of cognitive science, population dynamics and fuzzy reasoning and fulfils the following goal: 
- the dynamic evolution of the population system consisting of these elementary school students is provided and the asymptotic behaviour of the system is found. It is thus verified that there is actually an important future concentration to the most scientific models.

\section{A Fuzzy Markov System describing the dynamic evolution of the system}

Let us first give a short description of a closed homogeneous Markov population system (Vassiliou [1982]). Consider a population which is stratified into classes (states) according to distinctive properties and let $S=\{1,2, \ldots, n\}$ be the state space of the respective system. Assume, moreover, that the states are exclusive and exhaustive, i.e. each member of the system may be in one and only one state at any given time. The population structure of the system at any given time $t$ is represented by the row vector $\mathbf{N}(t)=\left[N_{1}(t), N_{2}(t), \ldots, N_{n}(t)\right]$, where $N_{i}(t)$ is the expected number of members of the system in state $i$ at time $t . T(t)$ denotes the total number of members in the system at time $t$ and $\Delta T(t)=T(t+1)-T(t)$. Let $\mathbf{P}$ denote the transition probability matrix of the system, i.e. $\mathbf{P}=\left\{p_{i j}\right\}_{i, j \in S}$, where:

$$
p_{i j}=\operatorname{prob}\{\text { a person moves to state } j / \text { the person was in state } i\} \text {. }
$$

The expected number of members in the various states at time $t$ is given by the following equation:

$$
\mathbf{N}(t)=\mathbf{N}(t-1) \mathbf{P} .
$$

One of the basic problems in the theory of NHMS and one of the main reasons for their impracticability is the uncertainty that is inherent in the estimation of the transition probabilities. More specifically, in order to apply the model of a closed homogeneous Markov system and to estimate the population structure of the system, the $(i, j)$-element of the matrix $\mathbf{P}$ must be determined, for every $i=1,2, \ldots, n$ and $j=1,2, \ldots, n$. This estimation is based on statistical data analysis, which can be accomplished whenever enough data is available. The above process obviously introduces uncertainty due to measurement errors and lack of data. This is the prime motivating factor for considering fuzzy logic and fuzzy reasoning in Markov systems. 
A reliable way to approximate the transition probabilities is to build a Fuгzy Inference System that can estimate the respective transition probabilities. More specifically, we apply fuzzy reasoning to the theory of Markov systems in order to estimate the transition probabilities and we use the method introduced in Symeonaki et al [2000, 2002]. The proposed method takes into consideration the heuristic knowledge that the experts of the system possess, without altering the stochastic property (the sum of the elements of each row is equal to 1) of the transition matrices.

In every population system there always exists a set of $m$ parameters $(b p 1, b p 2, \ldots, b p m)$, called the basic parameters of the system, which determine the population parameters of the system $(p p 1, p p 2, \ldots, p p l)$. These population parameters are used in order to estimate the transition probabilities, in the sense that each transition probability $p_{i j}(t)$ is a function of the population parameters of the system:

$$
p_{i j}(t)=f_{i j}(p p 1, p p 2, \ldots, p p l)
$$

where:

$$
\sum_{j \in S} f_{i j}(p p 1, p p 2, \ldots, p p l)=1,
$$

for any value of the parameters $p p 1, p p 2, \ldots, p p l$.

We will use the Fuzzy Inference System in order to find the population parameters of the system (Figure 1).

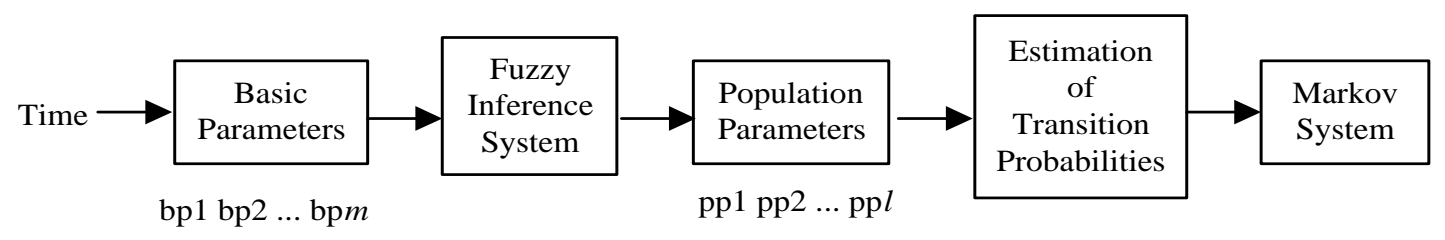

Figure 1. The structure of the Fuzzy Markov System.

Now observe that the mental model being used each time depends, among others, on the question being posed. Therefore, we look into each question separately, since both the state in which each student is (i.e. the mental model being used) and the transition from one state to the following states depend on the questions. 


\section{- Analysis of the $1^{\text {st }}$ Question}

The transitions between the mental models that took place in the first question are the following:

1. $\mathrm{A} 1 \rightarrow \mathrm{AP}, \mathrm{A} 4, \mathrm{P}+\mathrm{AP}, \mathrm{P}$ (6 times).

2. $\mathrm{A} 2 \rightarrow-$.

3. $\mathrm{A} 3 \rightarrow-$.

4. $\mathrm{A} 4 \rightarrow \mathrm{A} 4, \mathrm{M} 4, \mathrm{P}$ (4 times).

5. $\mathrm{M} 1 \rightarrow \mathrm{M} 1$ (5 times), P (3 times), A1.

6. $\mathrm{M} 4 \rightarrow \mathrm{P}$ (7 times), $\mathrm{P}+\mathrm{AP}$ (twice), $\mathrm{AP}, \mathrm{A} 1, \mathrm{M} 4$ (twice).

7. $\mathrm{P} \rightarrow \mathrm{P}$ (7 times), F (twice), O, M1.

8. $\mathrm{P}+\mathrm{AP} \rightarrow-$.

9. $\mathrm{AP} \rightarrow-$.

10. $\mathrm{F} \rightarrow-$.

11. $\mathrm{P} \rightarrow \mathrm{O}$,

were $\mathrm{O}$ denotes an unrecognisable state (i.e. the student used an unrecognisable mental model).

We now estimate the relative transition probabilities:

$$
\begin{aligned}
& p_{a_{1} a_{4}}=\frac{1}{9}, p_{a_{1} p}=\frac{6}{9}, p_{a_{1} p+a p}=\frac{1}{9}, p_{a_{1} a p}=\frac{1}{9}, \\
& p_{a_{4} a_{4}}=\frac{1}{6}, p_{a_{4} m_{4}}=\frac{1}{6}, p_{a_{4} p}=\frac{4}{6}, \\
& p_{m_{1} a_{1}}=\frac{1}{9}, p_{m_{1} p}=\frac{3}{9}, p_{m_{1} m_{1}}=\frac{5}{9}, \\
& p_{m_{4} a_{1}}=\frac{1}{13}, p_{m_{4} p}=\frac{7}{13}, p_{m_{4} m_{4}}=\frac{2}{13}, p_{m_{4} p+a p}=\frac{1}{13}, p_{m_{4} a p}=\frac{2}{13}, \\
& p_{p p}=\frac{7}{11}, p_{p f}=\frac{2}{11}, p_{p o}=\frac{1}{11}, p_{p m_{1}}=\frac{1}{11} .
\end{aligned}
$$

From the previous analysis we notice that there is a number of transitions that do not take place (e.g.: A4 $\rightarrow$ A1, A1 $\rightarrow$ A3, A1 $\rightarrow$ A2, etc). Moreover, from the mental models that fall into the category A only the mental model A4 is converted into a M model (M4). In addition, the initial population structure of the system is equal to: 
$\mathbf{N}(0)=\left[\begin{array}{lllllllll}9 & 0 & 0 & 6 & 9 & 13 & 11 & 0 & 0\end{array}\right]$. At this point we must stress that there is no data concerning a number of transition probabilities.

\section{- Analysis of the $2^{\text {nd }}$ Question}

The transitions that took place in the second question are:

1. $\mathrm{A} 1 \rightarrow \mathrm{A} 3, \mathrm{~A} 1$ (20 times), $\mathrm{A}+\mathrm{P}$ (5 times), F.

2. A2 $\rightarrow$ A1 (twice).

3. $\mathrm{A} 3 \rightarrow-$.

4. $\mathrm{A} 4 \rightarrow \mathrm{A} 3, \mathrm{~A} 2$.

5. $\mathrm{M} 1 \rightarrow \mathrm{A} 1$ (3 times), A3.

6. M4 $\rightarrow$ A1 (6 times), O, M4 (4 times).

7. $\mathrm{P} \rightarrow \mathrm{A} 1$.

The respective transition probabilities are estimated to be equal to:

$p_{a_{1} a_{3}}=\frac{1}{27}, p_{a_{1} a_{1}}=\frac{20}{27}, p_{a_{1} a+p}=\frac{5}{27}, p_{a_{1} f}=\frac{1}{27}$,

$p_{a_{4} a_{3}}=\frac{1}{2}, p_{a_{4} a_{2}}=\frac{1}{2}$

$p_{m_{1} a_{1}}=\frac{3}{4}, p_{m_{1} a_{3}}=\frac{1}{4}$

$p_{m_{4} a_{1}}=\frac{6}{11}, p_{m_{4} \mathrm{O}}=\frac{1}{11}, p_{m_{4} m_{4}}=\frac{4}{11}$,

$p_{p a_{1}}=1$,

$p_{a_{2} a_{1}}=1$.

Observe that:

The initial population structure of the system is:

$$
\mathbf{N}(0)=\left[\begin{array}{lllllllll}
27 & 2 & 0 & 2 & 4 & 11 & 1 & 0 & 2
\end{array}\right] .
$$

\section{- Analysis of the $3^{\text {rd }}$ Question}

The transitions that took place in the last question are:

1. A1 $\rightarrow$ A3 (3 times), A1 (32 times), A4.

2. $\mathrm{A} 2 \rightarrow-$.

3. $\mathrm{A} 3 \rightarrow \mathrm{A} 1$. 
4. A4 $\rightarrow$ A1 (3 times), A3, M4, A4.

5. $\mathrm{M} 1 \rightarrow-$.

6. M4 $\rightarrow$ A1 (twice).

7. $\mathrm{P} \rightarrow \mathrm{F}$ (twice).

8. $\mathrm{F} \rightarrow-$.

9. $\mathrm{O} \rightarrow-$.

It follows that the transition probabilities are equal to:

$$
\begin{aligned}
& p_{a_{1} a_{4}}=\frac{1}{36}, p_{a_{1} a_{3}}=\frac{3}{36}, p_{a_{1} a_{1}}=\frac{32}{36}, \\
& p_{a_{4} a_{4}}=\frac{1}{6}, p_{a_{4} m_{4}}=\frac{1}{6}, p_{a_{4} a_{1}}=\frac{3}{6}, p_{a_{4} a_{3}}=\frac{1}{6}, \\
& p_{m_{4} a_{1}}=1, \\
& p_{p f}=1, \\
& p_{a_{2} a_{1}}=1 .
\end{aligned}
$$

The initial population structure of the system is given by:

$$
\mathbf{N}(0)=\left[\begin{array}{lllllllll}
36 & 0 & 1 & 6 & 0 & 2 & 1 & 0 & 0
\end{array}\right] .
$$

In order to apply the model of a fuzzy Markov system it is of high importance first to examine which factors influence the transition probabilities. Notice, for example, that the number of lectures given in the crucial interval of these eight weeks plays an important role. The number of lectures result to a growth of the transition probabilities from the most naive mental models (the $\mathrm{M}$ models, for example) to the most advanced-scientific models (such as the A models, P, etc). Therefore:

- When we give more lectures on the concept of force (IF the NUMBER OF LECTURES is BIG), then the transition probability from the mental model M1 to $\mathrm{P}$ increases (since we have an increase of the rate of change of the transition probability from M1 to $\mathrm{P})$.

- When we give more lectures on the concept of force, then the transition probability from the mental model M1 to M1 decreases (since the rate of change of the transition probability from M1 to M1 decreases).

We assume that the factors (the population parameters) that influence the transition probabilities are the following: 
1. The number of lectures (hours) that the students attend in the period of these 8 weeks concerning the concept of force. We assume that the number of lectures range from 0 to 40 .

2. The student's efficiency. We assume that the student's efficiency takes values from 0 to 100 and that it depends upon a number of basic parameters (e.g. the teacher's communicability).

We define the fuzzy partitions $A_{1}$ and $A_{2}$ on the domains of the two factors respectively (apparently we do not know the exact output for an exact input) and on the domain of the outputs for example $p_{m_{1} m_{4}}, p_{m_{1} a_{1}}, B_{1}$ and $B_{2}$, respectively.

The fuzzy partitions are linguistic representations of their universe of discourse, therefore their elements are linguistic terms like "Low", "Big”, "Quite or Less Medium", etc. The relationship of the crisp universe of discourses is represented using linguistic rules that define a mapping of the fuzzy partition $A_{i}$ to the fuzzy partition $B_{j}$. This mapping is said to be a fuzzy association and represents the empirical, linguistic rules.

As far as the elements of $A_{i}$ and the elements of $B_{j}$ have a linguistic meaning, heuristic or empirical linguistic rules can be used in order to describe the input-output relationship. We concentrate the heuristic knowledge of the experts of the system in rules that have the following form:

1. When the NUMBER OF LECTURES is BIG, then $p_{m_{1} m_{4}}$ is SMALL.

2. When the NUMBER OF LECTURES is BIG, then $p_{m_{1} a_{1}}$ is BIG.

3. When the NUMBER OF LECTURES is SMALL, then $p_{m_{1} a_{1}}$ is SMALL.

4. When the STUDENT'S EFICIENCY is BIG, then $p_{m_{1} a_{1}}$ is BIG, etc.

Combining these rules with the well-known generalized modus ponens (GMP) rule of inference (Klir and Yuan [1996], Stamou and Tzafestas [1999]), the multi-conditional approximate reasoning schema (system rules) can be formulated as illustrated in Figures 2 and 3.

We denote by $w_{1}$ the degree in which the rule $i$ fires. Each rule corresponds to a matrix $\mathbf{P}_{i}$ and it can easily be proved by induction that if we use as $t$-norm the product, then $\sum_{i=1}^{k} w_{i}=1$. 
Therefore the transition matrix is of the form:

$$
\mathbf{P}=\sum_{i=1}^{k} w_{i} \mathbf{P}_{i}
$$

with:

$$
\mathbf{P}_{i} \mathbf{1}^{\prime}=\mathbf{1}^{\prime} \text { where } \mathbf{1}^{\prime}=[1,1, \ldots, 1]^{\prime} .
$$

We assume at this point that the matrices $\mathbf{P}_{i},(i=1,2, \ldots, k)$ are irreducible, regular, stochastic matrices.

The question that now rises from the previous analysis is what the asymptotic population structure of the system will be. In a system like the one we have just described it is true that the asymptotic population of the system is given by the following equation (Theorem 1, Symeonaki et al [2002]):

$$
\lim _{t \rightarrow \infty} \mathbf{N}(t)=\mathbf{N}(\infty)=T \mathbf{e}_{i}\left[\mathbf{I}-\left(\mathbf{I}-\sum_{i=1}^{k} w_{i} \mathbf{P}_{i}\right)\left(\mathbf{I}-\sum_{i=1}^{k} w_{i} \mathbf{P}_{i}\right)^{\#}\right] .
$$

Therefore, the asymptotic population structure of the system for each question is respectively equal to:

$$
\begin{aligned}
& \mathbf{N}_{1}(\infty)=\left[\begin{array}{lllllllll}
0.8114 & 0.3377 & 5.1611 & 0.3345 & 21.7903 & 4.054 & 5.9834 & 7.890 & 2.2658
\end{array}\right] \\
& \mathbf{N}_{2}(\infty)=\left[\begin{array}{lllllllll}
18.714 & 0.1357 & 0.9611 & 0.4349 & 1.7903 & 5.0254 & 7.892 & 8.797 & 5.2496
\end{array}\right] \\
& \mathbf{N}_{3}(\infty)=\left[\begin{array}{lllllllll}
0 & 0 & 0 & 0 & 0 & 0 & 16.555 & 14.355 & 2.2
\end{array}\right] .
\end{aligned}
$$

From these asymptotic population structures a future concentration to the scientific models is verified. This concentration actually happens quite fast, since it is easily proved that the rate of convergence of the population structure to its limit is geometric (Symeonaki and Stamou [2002]). 


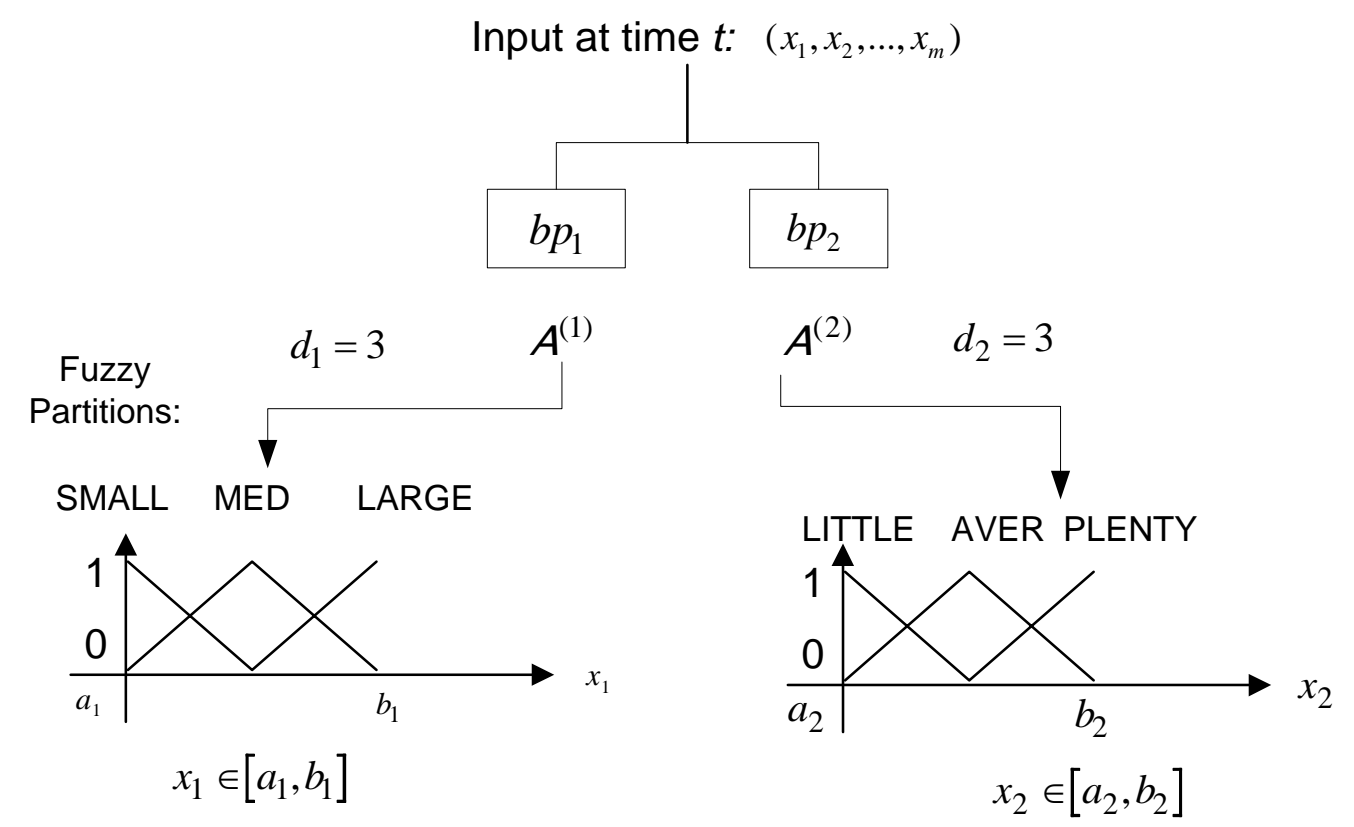

SYSTEM RULE FOR THE POPULATION PARAMETER $p p_{1}$ $1^{\text {st }}$ RULE: IF $\left(x_{1}, x_{2}\right)$ IS (SMALL, LITTLE), THEN $y_{1}$ IS LOW $2^{\text {nd }}$ RULE: IF $\left(x_{1}, x_{2}\right)$ IS (SMALL, AVER), THEN $y_{1}$ IS LOW $3^{\text {rd }}$ RULE: IF $\left(x_{1}, x_{2}\right)$ IS (SMALL, PLENTY), THEN $y_{1}$ IS AVER $4^{\text {th }}$ RULE: IF $\left(x_{1}, x_{2}\right)$ IS (MED, LITTLE), THEN $y_{1}$ IS LOW $5^{\text {th }}$ RULE: IF $\left(x_{1}, x_{2}\right)$ IS (MED, AVER), THEN $y_{1}$ IS AVER $6^{\text {th }}$ RULE: IF $\left(x_{1}, x_{2}\right)$ IS (MED, PLENTY), THEN $y_{1}$ IS HIGH $7^{\text {th }}$ RULE: IF $\left(x_{1}, x_{2}\right)$ IS (LARGE,LITTLE), THEN $y_{1}$ IS AVER $8^{\text {th }}$ RULE: IF $\left(x_{1}, x_{2}\right)$ IS (LARGE, AVER), THEN $y_{1}$ IS AVER $9^{\text {th }}$ RULE: IF $\left(x_{1}, x_{2}\right)$ IS (LARGE, PLENTY), THEN $y_{1}$ IS HIGH LOW AVER HIGH

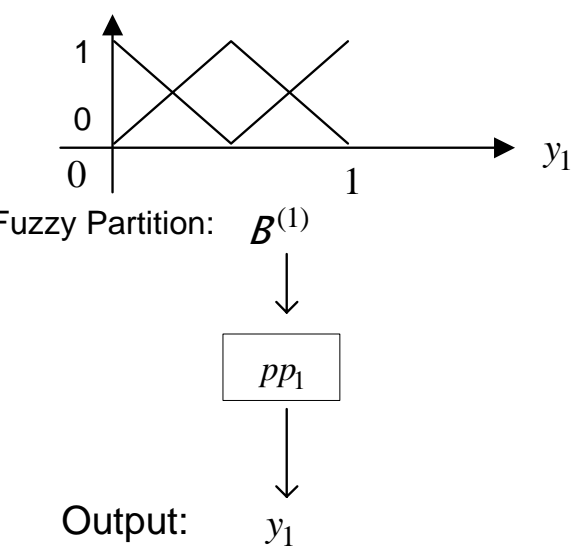

Figure 2: Regulations of the system for the population parameter $p p_{1}$ 


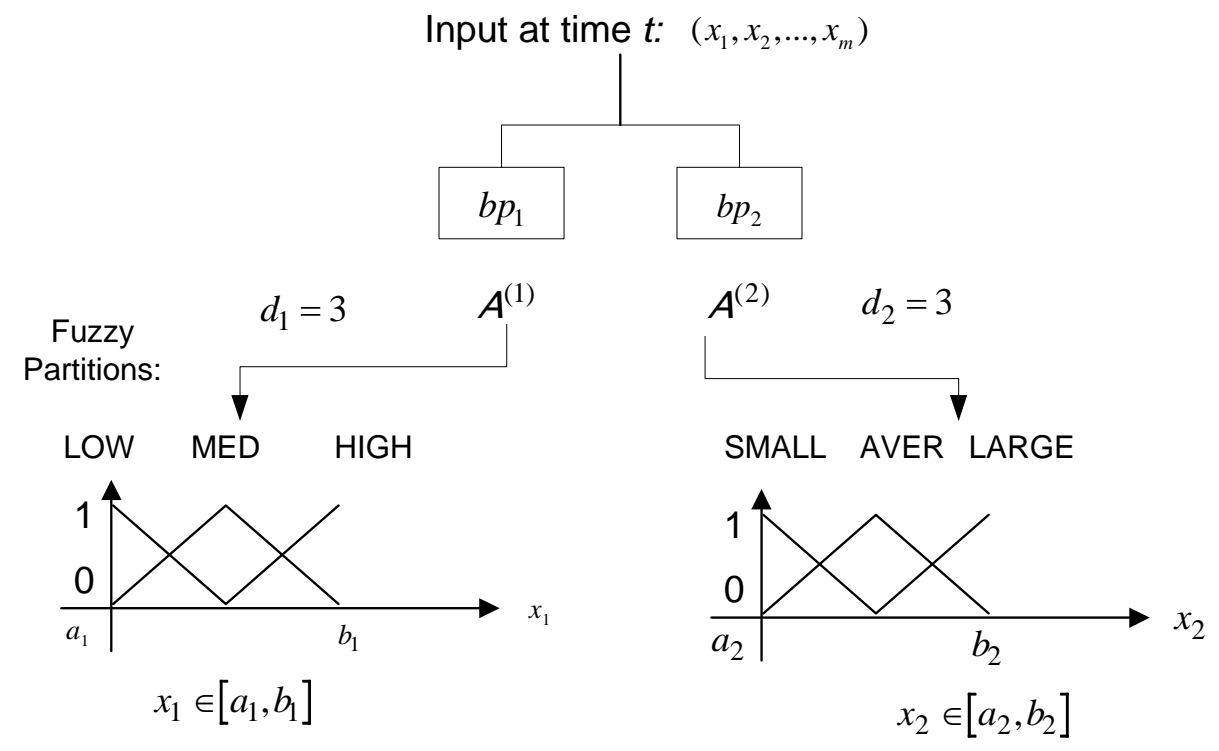

SYSTEM RULE FOR THE POPULATION PARAMETER $p p_{2}$ $1^{\text {st }}$ RULE: IF $\left(x_{1}, x_{2}\right)$ IS (LOW, SMALL), THEN $y_{1}$ IS SMALL $2^{\text {nd }}$ RULE: IF $\left(x_{1}, x_{2}\right)$ IS (LOW, MED), THEN $y_{1}$ IS SMALL $3^{\text {rd }}$ RULE: IF $\left(x_{1}, x_{2}\right)$ IS (LOW,LARGE), THEN $y_{1}$ IS MED $4^{\text {th }}$ RULE: IF $\left(x_{1}, x_{2}\right)$ IS $(M E D, S M A L L)$, THEN $y_{1}$ IS SMALL $5^{\text {th }}$ RULE: IF $\left(x_{1}, x_{2}\right)$ IS $(M E D, M E D)$, THEN $y_{1}$ IS MED $6^{\text {th }}$ RULE: IF $\left(x_{1}, x_{2}\right)$ IS (MED, LARGE), THEN $y_{1}$ IS LARGE $7^{\text {th }}$ RULE: IF $\left(x_{1}, x_{2}\right)$ IS $(H I G H, S M A L L)$, THEN $y_{1}$ IS MED $8^{\text {th }}$ RULE: IF $\left(x_{1}, x_{2}\right)$ IS (HIGH, MED), THEN $y_{1}$ IS MED $9^{\text {th }}$ RULE: IF $\left(x_{1}, x_{2}\right)$ IS (HIGH,LARGE), THEN $y_{1}$ IS LARGE

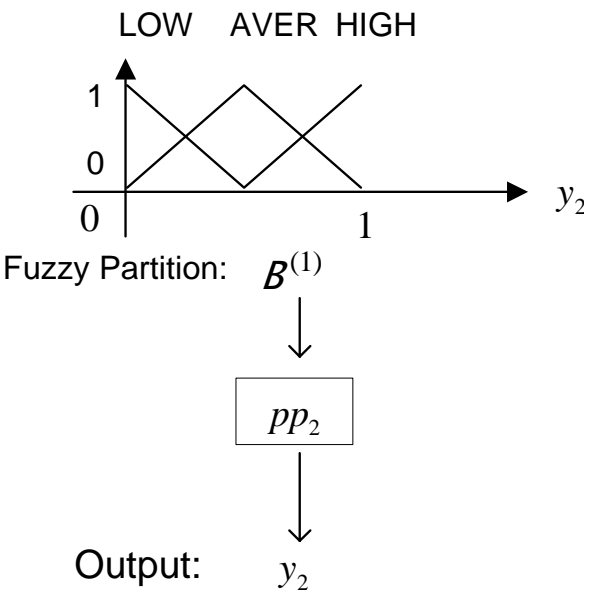

Figure 3: Regulations of the system for the population parameter $p p_{2}$. 


\section{References}

1. Carey S. [1991], Knowledge acquisition-enactment or conceptual change? In S. Carey, and R. Gelman (Eds.), The epigenesis of mind: Essays on Biology and Cognition, Hillsdale, NJ: Erlbaum.

2. Clement J. [1983], Students' preconceptions in introductory mechanics, American Journal of Physics, 50(1), 66-71.

3. Ioannides C. and S. Vosniadou [1993], Mental models of Force, $5^{\text {th }}$ European Conference for Research on Learning and Instruction, Aix - En - Provence, France.

4. Mc Closkey M. [1983], Naïve theories of motion, In: D. Genter \& A. L. Stevens (Eds), Mental models, Hillsdale, NJ: Erlbaum.

5. Morik M. [1996], A developmental case study on sequential learning: The day and night circle, In: Reimann, P. and Spada, H. editors, Learning in Humans and Machines Towards an Interdisciplinary Learning Science, chapter 12, pages 212-227, Elsevier.

6. Mühlenbrock K. [1994a], Computational models of learning in astronomy, Research Report 11, University of Dortmund, Lehrstuhl Informatik VIII.

7. Mühlenbrock K. [1994b], Menschliches and Maschinelles Lernen-Bildung und Revision von Begriffsstrukturen zum Tag/Nachtzyklus, Diplomarbeit, University of Dortmund, Fachbereich Informatik, Lehrstuhl VIII.

8. Symeonaki, M.A., Stamou, G. B. and Tzafestas S. [2000], Fuzzy Markov systems for the description and control of population dynamics, In: S. Trafestas (Ed.) Computational Intelligence in Systems and Control Design and Applications, Series: Advances in Intelligent Systems, p. 301-310, Kluwer Academic Publishers.

9. Symeonaki M.A., G.B. Stamou and S.G. Tzafestas [2002], Fuzzy Non-homogeneous Markov Systems, Applied Intelligence, 17(2): 203-214.

10. Vassiliou P.-C. G., Asymptotic behaviour of Markov systems [1982], J. Appl. Prob. 19 851-857.

11. Vosniadou S. [1994], Capturing and modelling the process of conceptual change, Learning and Instruction, 4, 45-69.

12. Vosniadou S. and W. Brewer [1992], Mental models of the earth: A study of conceptual change in childhood, Cognitive Psychology, 24, 535-585. 
13. Vosniadou S. and W. Brewer [1994], Mental models of the day/night circle, Cognitive Science, 18, 123-183.

14. Vosniadou S., M. Champesme, D. Kasyer et al. [2000], A psychological process model of the solution of mechanics problems by elementary school students: an interdisciplinary project, 1096-1101.

Received: September 2, 2005 